\title{
Spontaneous splenic rupture secondary to dabigatran: the last in a series of unfortunate events
}

\author{
Authors: Rachel Carey ${ }^{A}$ and Varun Nelatur ${ }^{B}$
}

\begin{abstract}
We present the case of a 77-year-old woman who had an accidental fall in her garden, resulting in a fracture of her left ankle. After manipulation of the fracture, she developed sudden onset shortness of breath. An echo led to the diagnosis of Takotsubo cardiomyopathy. Shortly after this she developed sudden onset receptive and expressive dysphasia. Magnetic resonance imaging (MRI) of the head confirmed a left parietal infarct thought to be secondary to left ventricular thrombus formation. She was started on dabigatran. A few days later, she developed abdominal pain, and was subsequently diagnosed with a spontaneous splenic rupture. This case was interesting due to the unusual chain of events following a simple fall, and also a rare complication of anticoagulant therapy.
\end{abstract}

KEYWORDS: Anticoagulation, splenic rupture, Takotsubo, thrombus, side effect

\section{Case presentation}

A previously well 77-year-old woman had an accidental fall in her garden, resulting in a left bimalleolar ankle fracture. After manipulation of the fracture in the emergency department, she developed sudden onset shortness of breath. A chest $X$-ray revealed acute pulmonary oedema, and she had a massive elevation in cardiac troponin $(400 \mathrm{ng} / \mathrm{L}$, later rising to $600 \mathrm{ng} / \mathrm{L})$.

An angiogram confirmed that she had normal coronary arteries and an echo demonstrated an akinetic apex, so she was diagnosed with Takotsubo cardiomyopathy. Two days later, she developed sudden onset receptive and expressive dysphasia. Magnetic resonance imaging (MRI) confirmed a left parietal infarct, thought to be embolic in nature secondary to the Takotsubo cardiomyopathy. Therefore, the patient was started on dabigatran.

The following day, she developed severe abdominal pain and her oxygen saturations began to fall. No obvious cause was found and so she was treated with analgesia and antibiotics in view of

Authors: ${ }^{\text {A }}$ core medical trainee, Royal Berkshire Hospital, Reading, UK; ${ }^{B}$ consultant in acute medicine and stroke medicine, Royal Berkshire Hospital, Reading, UK raised inflammatory markers. Three days later, she had ongoing abdominal pain and her haemoglobin began to drop. Eventually computed tomography (CT) abdomen and pelvis revealed a spontaneous splenic rupture (Fig 1).

\section{Diagnosis}

Sudden onset pulmonary oedema with a raised troponin following an acute stressor (ankle fracture manipulation in our case) is very suggestive of Takotsubo cardiomyopathy. Coronary artery disease was excluded with angiography, and subsequent echocardiography confirmed the diagnosis in our case.

Cardioembolic stroke due to the formation of a left ventricular (LV) thrombus is a rare but well-documented complication of Takotsubo syndrome. Embolic stroke secondary to angiography was also considered but tends to be more immediate following the procedure. Dabigatran was started to prevent further embolic events.

The following day, the patient developed severe upper abdominal pain with shortness of breath, an oxygen requirement and raised inflammatory markers. She was high risk for pulmonary embolism given her ankle fracture and immobility, but this was rapidly ruled out with a CT pulmonary angiogram (CTPA). Hospital-acquired pneumonia was also a differential, and she was treated empirically with antibiotics to cover for her chest, but again this did not fit with her normal chest X-ray. Abdominal causes such as pancreatitis, bowel obstruction and biliary sepsis were also considered, but normal abdominal X-ray, liver function tests, amylase and lactate made these less likely.

Her inflammatory markers did not improve and her symptoms went largely unexplained for the next few days until a drop in haemoglobin was noted. Following this, a CT abdomen and pelvis confirmed a spontaneous splenic rupture.

\section{Initial management and prognosis}

After the diagnosis of splenic rupture was confirmed, dabigatran was held, she was transfused and given praxbind.

\section{Case progression and outcome}

With a few weeks of speech and language therapy input on the Acute Stroke Unit, the patient recovered well from her parietal infarct, being left with only mild difficulty with writing.

Following conservative management of her splenic rupture, her haemoglobin stabilised and her abdominal pain resolved. 
(a)

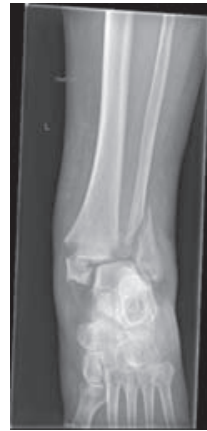

(b)

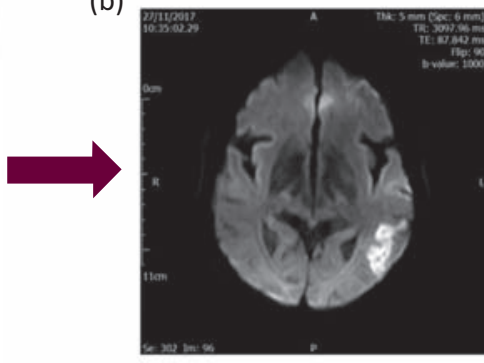

(c)

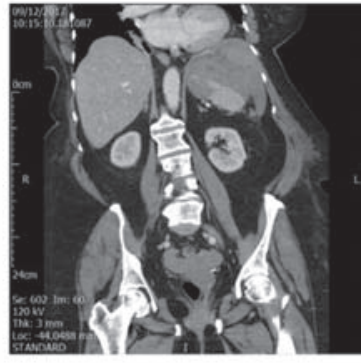

Fig 1. The series of unfortunate events. (a) shows a plain radiograph showing the initial insult - the bimalleolar ankle fracture, (b) shows an MRI head demonstrating a parietal infarct, (c) shows a CT with contrast demonstrating an area of haemorrhage within the spleen. $\mathrm{CT}=$ computed tomography: MRI = magnetic resonance imaging
After discussion with the haematology team, she was cautiously started on prophylactic dose tinzaparin with close haemoglobin monitoring, as she remained high risk of further arterial and venous thromboembolism. Her haemoglobin remained stable and she was eventually discharged to a rehabilitation hospital.

The main longstanding issue is, surprisingly, the patient's ankle fracture. Surgical fixation would have been the optimal management; however, she had been deemed too high risk for surgery in the acute phase due to multiple medical complications, and so has required prolonged immobilisation of the fracture and close orthopaedic follow-up.

\section{Discussion}

Takotsubo cardiomyopathy is characterised by sudden akinesia and ballooning of the cardiac apex, often triggered by an acute stressor. Although the process is reversible, it can be associated with some significant complications, such as arrhythmias, acute pulmonary oedema, left ventricular (LV) thrombus formation and subsequent thromboembolism. Incidence between studies is variable, but a recent paper estimated the incidence of LV thrombus formation following Takotsubo syndrome to be around $2.2 \%$, with $17 \%$ of those going on to develop a stroke. ${ }^{1,2}$ There are currently no guidelines regarding anticoagulation in Takotsubo patients; however, those who do have thromboembolic events, or a proven LV thrombus on echo, are generally started on anticoagulation. It has been suggested that it is reasonable to consider prophylactic anticoagulation in Takotsubo patients who are at 'high risk' of LV thrombus formation (post-menopausal females, raised troponin, apical ballooning on echocardiography) ${ }^{2}$, but more studies are required to investigate which patients are most likely to benefit from prophylactic anticoagulation, and whether prevention of stroke will outweigh the bleeding risks in this patient group.

Splenic rupture is most commonly associated with trauma and spontaneous ruptures are more frequently seen in patients with abnormal spleens (such as those with haematological malignancy or infection). However, there are a few case reports of spontaneous ruptures secondary to anticoagulation alone. ${ }^{3,4}$ In one systematic review, it was estimated that only $9.1 \%$ of spontaneous ruptures were drug or treatment related, ${ }^{5}$ however, more recent papers have estimated the incidence to be higher. Clues to the diagnosis include left-sided abdominal pain that may be referred to the shoulder, falling haemoglobin levels and features of shock. However, our patient did not exhibit these signs until 3 days after the initial abdominal pain, so the presentation can be more vague in some cases. Conservative management is usually adequate, but surgical intervention should be considered when patients become haemodynamically compromised.

This case was unusual due to the multiple complications that occurred following a simple mechanical fall. It also raised some important issues surrounding anticoagulation in Takotsubo syndrome and difficulty in diagnosing a spontaneous splenic rupture.

\section{Key learning points}

> Thromboembolic stroke is a rare complication of takotsubo cardiomyopathy. Anticoagulation should be considered to prevent further embolic events in these patients

> Spontaneous splenic rupture should be considered as a rare cause of abdominal pain in patients on anticoagulants. Other clues may include left shoulder pain, drop in haemoglobin, raised inflammatory markers and shock

> Management of splenic rupture is usually supportive - with reversal of anticoagulation and blood products, but surgical management or embolisation should be considered in those with haemodynamic compromise

> In patients with symptoms that remain unexplained despite initial investigations, it is important to continue strive to find an explanation.

\section{Consent}

Written informed consent was obtained from the patient for the publication of this case report and the clinical images.

\section{Author contributions}

The patient was assessed by VN and RC, the case written up by RC with elements of case presentation and discussion revised by VN.

\section{References}

1 Heckle M, McCoy C, Akinseye O, Khouzam R. Stress-induced thrombus: prevalence of thromboembolic events and the role of anticoagulation in Takotsubo cardiomyopathy. Ann Transl Med 2018;6:4.

2 Santoro F, Stiermaier T, Tarantino $\mathrm{N}$ et al. Left ventricular thrombi in Takotsubo syndrome: incidence, predictors, and management: results from the GEIST (German Italian Stress Cardiomyopathy) Registry. J Am Heart Assoc 2017;6:e006990.

3 Kubber M, Kroft L, de Groot B. Non-traumatic splenic rupture in a patient on oral anticoagulation. Int J Emerg Med 2013;6:16. 
4 Lowry L, Goldner J. Spontaneous splenic rupture associated with apixaban: a case report. J Med Case Rep 2016;10:217.

5 Renzulli P, Hostettler A, Schoepfer AM, Gloor B, Candinas D. Systematic review of atraumatic splenic rupture. $\mathrm{Br}$ ] Surg 2009:96:1114-21.
Address for correspondence: Dr Rachel Carey, Royal Berkshire Hospital, Acute Stroke Unit, 21 Craven Road, Reading RG1 5LE, UK.

Email: racheljcarey@outlook.com

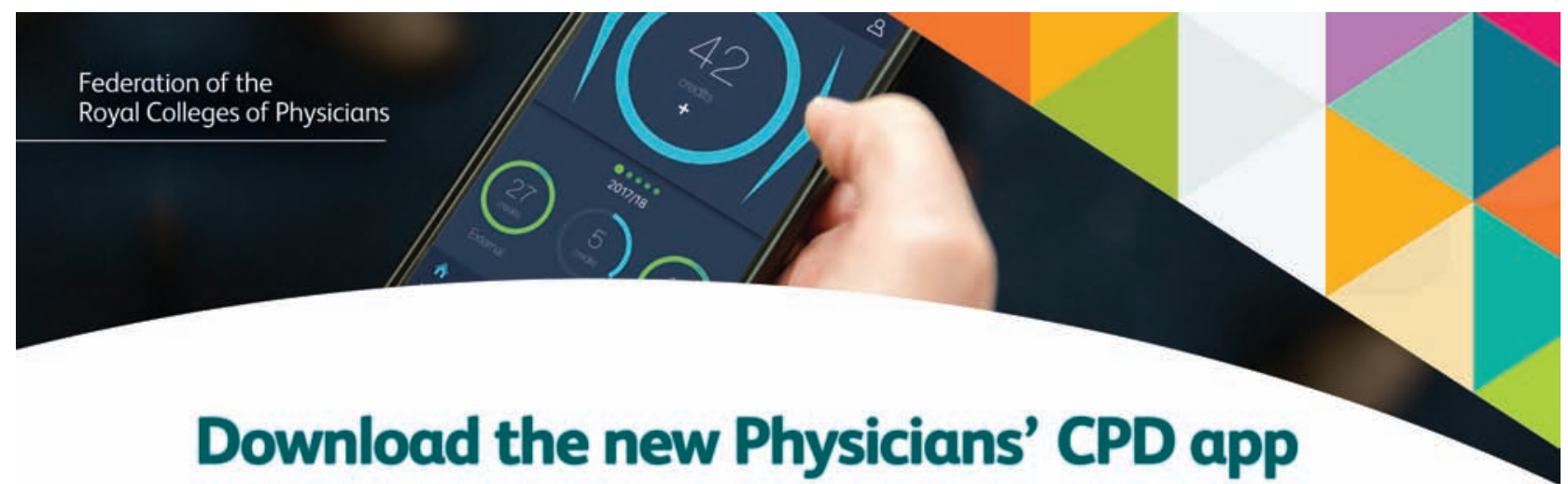
Record and reflect on the go

> Add and reflect on your educational activities, wherever you are your changes will sync automatically with your online CPD diary"

$>$ Voice transcribe your development needs and reflections instantly

$>$ Access our list of over 5.000 approved activities

$>$ Keep track of your CPD and monitor your progress

to achieving your annual credit requirements

'An excellent and user-friendly app. I shall be using my CPD diary more frequently now!'

- Dr Andrew Lansdown, Consultant Endocrinologist

Simply download the Physicians' CPD app on your smartphone or tablet today and log in using your CPD diary account details. Now avallable on the iOS App Store and the Google Play Store
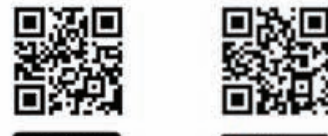

A App Store

$D$ coosteptar

Physicians' CPD

https://cpd.rcplondon.ac.uk 